ELSEVIER

\title{
Preoperative Cardiac Assessment in Patients Undergoing Aortic Surgery: Analysis of Factors Affecting the Cardiac Outcomes
}

\author{
Nicola Troisi, ${ }^{1}$ Walter Dorigo, ${ }^{1}$ Patrizia Lo Sapio, ${ }^{2}$ Giovanni Pratesi, ${ }^{3}$ Raffaele Pulli, ${ }^{1}$ \\ Gian Franco Gensini, ${ }^{2}$ and Carlo Pratesi, ${ }^{1}$ Florence and Rome, Italy
}

\begin{abstract}
Background: The purpose of this study was to evaluate the effectiveness of a standardized preoperative cardiac assessment in reducing the rate of perioperative cardiac complications in patients undergoing aortic surgery, paying particular attention to the analysis of the factors affecting perioperative cardiac outcomes.

Methods: Between January 2005 and August 2008, a total of 531 elective interventions for abdominal aortic aneurysms were performed at our institution. All patients underwent preoperative evaluation on an outpatient basis, which included a standardized cardiac assessment protocol to evaluate perioperative cardiac risk. A surgical (open or endovascular) intervention was performed in all patients and perioperative ( $<30$ days) results in terms of overall and cardiac mortality and morbidity rates were recorded.

Results: Preoperative electrocardiogram, transthoracic echocardiography, and cardiology consultation were performed in all patients. In 348 cases (65.5\%), further evaluation of cardiac functional capacity with noninvasive stress testing was recommended. A positive noninvasive stress test was noted in $86(24.7 \%)$ of 348 patients and coronary angiography was performed in $34(39.5 \%)$ of 86 patients. Thirteen of these patients underwent successful coronary revascularization (11 percutaneous transluminal coronary angioplasty, two coronary artery bypass graft) before vascular surgery. Thirty-day overall rate and cardiac mortality rate were $1.1 \%$ and $0.6 \%$, respectively, and 30 -day overall and cardiac morbidity rates were $11.5 \%$ and $6.8 \%$, respectively. Univariate analysis demonstrated that in patients aged $>80$ years, chronic renal failure, congestive heart failure, valvular heart disease, positive noninvasive stress testing, and open surgical treatment significantly affected 30-day cardiac morbidity; however, only valvular heart disease and positive noninvasive stress testing maintained their significance with multivariate analysis ( $p=0.005 ; 95 \% \mathrm{Cl}, 1.6-14.4$; and $p=0.02 ; 95 \% \mathrm{Cl}, 1.2-8.1$, respectively). None of the examined factors significantly affected 30 -day cardiac mortality.

Conclusion: In our experience, the use of a routine preoperative cardiac assessment allowed us to obtain satisfactory perioperative results in patients undergoing abdominal aortic surgery. Very few patients, however, require cardiac revascularization before abdominal aortic surgery. Patients with positive stress test may benefit from an endovascular treatment if anatomically feasible.
\end{abstract}

\section{INTRODUCTION}

Cardiac complications (myocardial infarction, acute congestive heart failure, fatal arrhythmias)

\footnotetext{
${ }^{I}$ Department of Vascular Surgery, University of Florence, Florence, Italy. Italy.

${ }^{2}$ Department of Heart and Vessels, University of Florence, Florence, Italy

${ }^{3}$ Department of Vascular Surgery, University of Tor Vergata, Rome,
}

represent the major cause of perioperative morbidity and mortality in patients undergoing vascular surgery. ${ }^{1-4}$ This is particularly related to the frequent association of systemic atherosclerosis

Correspondence to: Nicola Troisi, Department of Vascular Surgery, University of Florence, Viale Morgagni 85, 50134, Florence, Italy, E-mail:nicola.troisi@alice.it

Ann Vasc Surg 2010; 24: 733-740

DOI: 10.1016/j.avsg.2010.01.009

(C) Annals of Vascular Surgery Inc.

Published online: May 17, 2010 
(carotid bifurcation, abdominal aorta, peripheral arteries) with coronary artery disease (CAD).$^{5,6}$ In fact, it has been demonstrated that a history of $\mathrm{CAD}$, congestive heart failure, diabetes mellitus, and chronic renal failure increases perioperative cardiac morbidity and mortality rates. ${ }^{7,8}$

To reduce perioperative cardiac complications in patients undergoing major vascular surgery, cardiac assessment consisting of electrocardiogram (ECG), echocardiography, cardiology consultation, and, in selected cases, noninvasive stress testing has been advocated by several authors. ${ }^{9}$ Noninvasive stress testing is widely used to assess the risk of cardiac complications in the perioperative period, even if its predictive power is controversial. ${ }^{10}$ Perioperative beta-blocker or statin medical therapy seems to be associated with a reduced risk of in-hospital death among patients undergoing major noncardiac surgery, ${ }^{9,11}$ even if recent large randomized studies did not strongly support these hyphoteses. ${ }^{12}$

At the moment, however, there is no strong data to support prophylactic surgical or endovascular myocardial revascularization in reducing perioperative cardiac risk after major vascular surgery. ${ }^{13}$ Godet et al. ${ }^{14}$ demonstrated that preoperative coronary intervention does not seem to significantly reduce the risk of cardiac complications in the perioperative period in patients undergoing aortic surgery. The recent American College of Cardiology/American Heart Association (ACC/AHA) guidelines ${ }^{9}$ stated that myocardial intervention is rarely necessary to simply lower the risk of vascular surgery unless such intervention is indicated, regardless of the patients' preoperative status. As a consequence, there is a general agreement that myocardial revascularization before vascular surgery should be reserved for patients with unstable angina or stable angina with significant (left main or 3-vessel) CAD. ${ }^{9}$

The purpose of this study was to evaluate the effectiveness of a standardized preoperative cardiac assessment in reducing the rate of perioperative cardiac complications in patients undergoing aortic surgery, paying particular attention to the analysis of the factors affecting perioperative cardiac outcomes.

\section{MATERIALS AND METHODS}

Between January 2005 and August 2008, a total of 531 elective interventions for abdominal aortic aneurysms (AAAs) were performed. All patients underwent preoperative evaluation on an outpatient basis which included a standardized cardiac assessment protocol. Data from the preoperative evaluations were prospectively collected in a dedicated database, which included demographic data, preoperative risk factors, and clinical and diagnostic assessment. All patients underwent surgical (open or endovascular) intervention and perioperative ( $<30$ days) results in terms of overall and cardiac morbidity, and mortality rates were recorded.

\section{Preoperative Diagnostic Assessment}

All patients preoperatively underwent an extensive assessment of clinical history and a physical examination, a two-view chest X-ray, an ECG, laboratory tests including complete blood count, coagulative parameters, and blood chemistries, and a Duplex ultrasound scanning of the aortoiliac and carotid arteries. Pulmonary functional capacity and response to bronchodilators were investigated and the presence of carbon dioxide retention through arterial blood gas analysis was evaluated in patients with history of chronic obstructive pulmonary disease or asthma, according to American College of Physicians guidelines. ${ }^{15}$

Regardless of the ECG findings, all patients underwent cardiology consultation and transthoracic echocardiography. Because most of the patients came from peripheral centers, the cardiologist recommended changes in medications so that the best medical treatment can be given to all the patients; statins and anti-platelet agents in all patients, and beta-blockers in selected cases.

Our series comprised patients undergoing aortic surgery. On the basis of ACC/AHA 2007 guidelines, ${ }^{9}$ patients undergoing open aortic surgery should be considered at high cardiac risk $(>5 \%)$ and patients undergoing endovascular aortic surgery should be considered at intermediate cardiac risk (1-5\%). The cardiologist performed the consultation before the surgeon decided the kind of treatment for the patient; hence, all patients were considered at high surgical risk and the decision to make further preoperative tests was taken by the cardiologist on the basis of history, physical examination, ECG, and echocardiographic anomalies. In particular, the cardiologist suggested a noninvasive stress testing in presence of past or recent ischemic heart disease, compensated or prior heart failure, past or recent cerebrovascular disease, diabetes mellitus, chronic renal failure (history), alterations at cardiac auscultation (physical examination), significant arrhythmias, abnormal Q waves (ECG anomalies), severe valvular diseases, abnormal areas of motility, and 
left ventricular ejection fraction $<40 \%$ (echocardiographic anomalies). All noninvasive stress tests were conducted after withdrawal of beta-blockers.

At this point, all patients underwent computed tomography angiography of entire thoracic and abdominal aorta to evaluate for the presence of other aortic lesions and to determine the kind of repair (assessment of feasibility for endovascular repair).

In patients with a negative cardiac stress test, surgery was performed without any other investigations. Patients with a positive stress test (a peak heart rate $<85 \%$ of the maximum predicted at the dobutamine stress echocardiography ${ }^{16}$ or reversible defects that involved $>20 \%$ of myocardial segments at the radionuclide myocardial perfusion imaging ${ }^{17}$ ) underwent new consultation and the cardiologist, also on the basis of surgeon's decision, proposed higher levels of postoperative care in the Intensive Care Unit (ICU) and further changes in medications with the adjunct of beta-blockers or suggested to perform a classical coronary angiography with possible therapeutic procedures (percutaneous transluminal coronary angioplasty [PTCA] or coronary artery bypass graft [CABG]). Coronary revascularization before vascular surgery was performed in patients who had unstable angina, stable angina with 3-vessel disease, or a significant stenosis of the left main coronary artery.

In these patients, the vascular intervention was consequently delayed. If PTCA was performed, patients received a single antiplatelet therapy (aspirin) for 4 weeks. If a stent was inserted, a dual antiplatelet therapy was administered (aspirin and clopidogrel) for 4 weeks (bare-metal coronary stents) or 6 months (drug-eluting coronary stents).

Our decisional algorithm is summarized in Fig. 1.

\section{Definition of Parameters}

Our database included demographic data, preoperative risk factors, and clinical and diagnostic assessment. Definitions of these parameters are shown in Table I.

\section{Statistical Analysis}

Statistical analysis was performed by SPSS 15.0 for Windows (SPSS Inc., Chicago, IL,). General characteristics, cardiovascular risk factors, results of preoperative diagnostic assessment, and different surgical approaches for perioperative $(<30$ days) cardiac mortality and morbidity rates were analyzed and compared by mean of $\chi^{2}$ test or Fisher test when necessary. Statistical significance was defined at $p<0.05$ level.

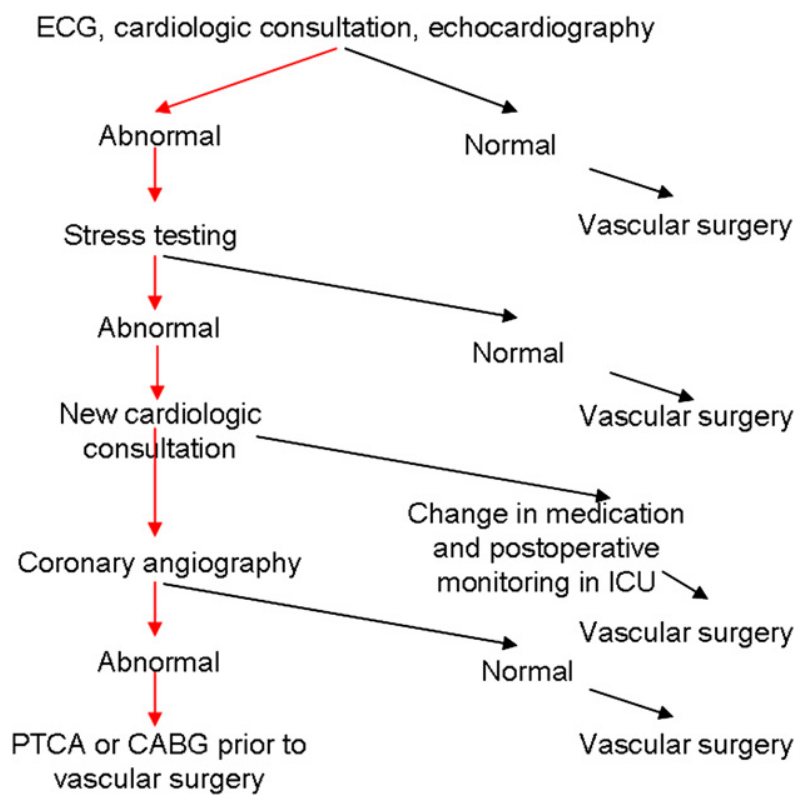

Fig. 1. Preoperative cardiac evaluation: our decisional algorithm.

Multivariate analysis (stepwise logistic regression analysis) was performed to identify independent predictors of overall and of cardiac death and complications.

\section{RESULTS}

\section{Study Group}

Patients were predominantly men $(474,89.3 \%)$, with a mean age of 73 years (range, 51-90). Demographic data, preoperative risk factors, and clinical assessment are shown in Table II.

\section{Preoperative Cardiac Assessment and Operative Management}

According to our decisional algorithm (Fig. 1), 183 patients $(34.5 \%)$ underwent aortic surgery without any further evaluations. The remaining 348 patients $(65.5 \%)$ underwent additional evaluation with dobutamine stress echocardiography (208 cases, $59.8 \%)$ or radionuclide myocardial perfusion imaging ( 140 cases, $40.2 \%$ ).

Of these 348 patients, $262(75.3 \%)$ had a negative stress test, and the remaining 86 patients $(24.7 \%)$ had a positive one. In $52(60.5 \%)$ of these 86 patients, consultant suggested beta-blocker medical therapy and postoperative ICU stay. In the remaining 34 patients $(39.5 \%)$ coronary angiography was performed; no significant CAD was noted in 21 cases and the patients proceeded to vascular surgery, whereas the remaining 13 patients had significant 
Table I. Parameters used to elaborate the perioperative cardiac risk

\begin{tabular}{|c|c|}
\hline Parameter & Definition \\
\hline Sex & Male/female \\
\hline Older age & $>80 \mathrm{yr}$ \\
\hline Smoking & Current or previous (within $10 \mathrm{yr}$ ) smoker \\
\hline Hypertension & $\begin{array}{l}\text { A systolic blood pressure of } 140 \mathrm{~mm} \mathrm{Hg} \text { or greater, a diastolic blood } \\
\text { pressure of } 90 \mathrm{~mm} \mathrm{Hg} \text { or greater, or use of antihypertensive } \\
\text { medications }\end{array}$ \\
\hline Hypercholesterolemia & $\begin{array}{l}\text { Total cholesterol serum level }>200 \mathrm{mg} / \mathrm{dL} \text { or low-density-lipoprotein } \\
\text { (LDL) cholesterol }>140 \mathrm{mg} / \mathrm{dL} \text { or use of statins }\end{array}$ \\
\hline Diabetes mellitus & $\begin{array}{l}\text { Fasting glucose of } 126 \mathrm{mg} / \mathrm{dL} \text { or greater, oral glucose tolerance test } \\
\text { (OGTT) of } 200 \mathrm{mg} / \mathrm{dL} \text { or greater, or use of oral antidiabetics/insulin }\end{array}$ \\
\hline Chronic obstructive pulmonary disease & $\begin{array}{l}\text { Chronic parenchymal X-ray changes or pulmonary function tests less } \\
\text { than } 80 \% \text { of predicted }\end{array}$ \\
\hline Chronic renal failure & Creatinine serum level greater than $2.0 \mathrm{mg} / \mathrm{dL}$ \\
\hline Coronary artery disease & $\begin{array}{l}\text { Documented history of at least one of the following: positive ECG } \\
\text { changes consistent with myocardial ischemia, angina, myocardial } \\
\text { infarction, PTCA, CABG }\end{array}$ \\
\hline Myocardial infarction & History of previous myocardial infarction \\
\hline PTCA & History of previous myocardial endovascular revascularization \\
\hline CABG & History of previous myocardial surgical revascularization \\
\hline Congestive heart failure & $\begin{array}{l}\text { Presence of any of the following: history of congestive heart failure, } \\
\text { pulmonary edema or paroxysmal nocturnal dyspnea, bilateral rales or } \\
\text { S3 gallop at physical examination, pulmonary vascular redistribution } \\
\text { at chest radiograph }\end{array}$ \\
\hline Valvular heart disease & $\begin{array}{l}\text { Presence of any of the following: echocardiographic features: aortic } \\
\text { stenosis with a maximal jet velocity } \geq 2.5 \mathrm{~m} / \mathrm{s} \text {, mitral stenosis with a } \\
\text { valve area } \leq 2 \mathrm{~cm}^{2} \text {, aortic regurgitation with a grade } \geq 2 / 4 \text {, mitral } \\
\text { regurgitation with a grade } \geq 2 / 4 \text {, patients who have undergone any } \\
\text { intervention on a cardiac valve }\end{array}$ \\
\hline Arrhythmia & $\begin{array}{l}\text { Presence of any of the following: atrial fibrillation, high grade } \\
\text { atrioventricular block, ventricular or supraventricular ectopy }\end{array}$ \\
\hline Implanted devices & $\begin{array}{l}\text { Presence of a pace-maker or a implantable cardioverter defibrillator } \\
\text { (ICD) }\end{array}$ \\
\hline Positive stress testing & $\begin{array}{l}\text { Poor cardiac functional capacity diagnosed at radionuclide myocardial } \\
\text { perfusion imaging or dobutamine stress echocardiography or treadmill } \\
\text { exercise test }\end{array}$ \\
\hline Cardiac treatment & PTCA or CABG prior to vascular surgery \\
\hline
\end{tabular}

coronary artery lesions and were successfully treated (11 PTCA, 2 coronary artery bypass graft) before vascular surgery (Table III).

Conventional open aneurysm repair with a transperitoneal approach was carried out in 298 (56.1\%) of 531 patients, whereas in the other 233 cases $(43.9 \%)$ the AAA was excluded through the implantation of an endoluminal graft. Overall, 144 patients $(27.1 \%)$ required a higher level of postoperative monitoring in the ICU (Table III).

In the perioperative period, a beta-blocker therapy was continued in all the patients receiving it before the vascular intervention, whereas in the remaining patients it was administered only in the perioperative period. Furthermore, regarding cardiac management the surveillance for perioperative myocardial infarction was obtained with the measurement of troponin $\mathrm{T}$ in the recovery room after operation and on the next two postoperative mornings; the troponin $\mathrm{T}$ monitoring was continued, if indicated. The diagnosis of myocardial infarction was determined by troponin $\mathrm{T}$ values combined with changes on either ECG or symptoms referred by the patient.

\section{Early Results ( <30 Days)}

In our series, 30-day overall mortality rate was 1.1\% (6/531 patients). Four deaths occurred in patients operated on with an open surgical approach: in three cases, a cardiac-related death (two acute congestive heart failure with pulmonary edema and one myocardial infarction) was identified, and in one patient the cause of death was a multiple 
Table II. Demographic data, preoperative risk factors, and clinical assessment

\begin{tabular}{lc}
\hline & Number of patients (\%) \\
\hline Male sex & $474(89.3 \%)$ \\
Median age $(\mathrm{yr})$ & 73 \\
Older age $(>80 \mathrm{yr})$ & $114(21.5 \%)$ \\
Smoking & $478(90 \%)$ \\
Hypertension & $377(71 \%)$ \\
Hypercholesterolemia & $207(39 \%)$ \\
Diabetes mellitus & $60(11.3 \%)$ \\
Chronic obstructive & $296(55.7 \%)$ \\
$\quad$ pulmonary disease & $50(9.4 \%)$ \\
Renal chronic failure & $161(30.3 \%)$ \\
Coronary artery disease & $99(18.6 \%)$ \\
Myocardial infarction & $58(10.9 \%)$ \\
PTCA & $37(7 \%)$ \\
CABG & $33(6.2 \%)$ \\
Congestive heart failure & $53(10 \%)$ \\
Valvular heart disease & $76(14.3 \%)$ \\
Arrhythmia & $16(3 \%)$ \\
Implanted devices &
\end{tabular}

Table III. Preoperative cardiac assessment and operative management

\begin{tabular}{lc}
\hline & Number of patients (\%) \\
\hline Stress testing & $348 / 531(65.5 \%)$ \\
Positive stress testing & $86 / 348(24.7 \%)$ \\
Coronary angiography & $34 / 86(39.5 \%)$ \\
PTCA & $11 / 34(32.4 \%)$ \\
CABG & $2 / 34(5.9 \%)$ \\
Endovascular treatment & $233 / 531(43.9 \%)$ \\
Postoperative ICU & $144 / 531(27.1 \%)$ \\
\hline
\end{tabular}

organ failure. In the endovascular subgroup, two patients died from acute ischemic colitis. One of the three cardiac deaths occurred in a patient who had undergone a preoperative coronary revascularization with PTCA. Overall for our series, 30-day cardiac mortality rate was $0.6 \%$ (3/531 patients) (Table IV). At univariate analysis, none of the examined factors significantly affected 30-day cardiac mortality.

At 30 days, 61 patients (11.5\%) developed 63 major complications, requiring a prolonged hospital stay or a reintervention: 36 had at least one cardiac complication (17 myocardial infarction requiring a primary PTCA in seven cases, 14 episodes of atrial fibrillation, and five acute congestive heart failure). Sixteen of the 17 perioperative myocardial infarctions occurred in untreated patients. Overall 30 -day cardiac morbidity rate was $6.8 \%$ (Table IV).
Table IV. Early results ( $<30$ days)

\begin{tabular}{lc}
\hline & Number of patients $(\%)$ \\
\hline Overall mortality & $6(1.1 \%)$ \\
& 3 cardiac \\
& 2 ischemic colitis \\
1 MOF & $(0.6 \%)$ \\
Cardiac mortality & 2 pulmonary edema \\
& 1 myocardial infarction \\
Overall morbidity & $(11.5 \%)$ \\
& 36 cardiac \\
& 7 bleeding \\
4 pancreatitis \\
3 ischemic colitis \\
3 acute lower limb ischemia \\
2 stroke \\
2 acute renal failure \\
$(1$ in dialysis $)$ \\
1 multiple organ \\
failure (MOF) \\
1 peripheral embolization \\
1 paraplegia \\
1 hemorrhagic gastritis \\
1 bowel obstruction \\
1 epileptic seizure \\
$36(6.8 \%)$ \\
17 myocardial infarction \\
14 atrial fibrillation \\
5 congestive heart failure \\
\hline Cardiac morbidity \\
\end{tabular}

Univariate analysis demonstrated that in patients aged $>80$ years, chronic renal failure, congestive heart failure, valvular heart disease, positive noninvasive stress testing, and open surgical treatment significantly affected 30-day cardiac morbidity. Valvular heart disease and positive noninvasive stress testing maintained their significance also with multivariate analysis $(p=0.005 ; 95 \%$ CI, 1.6-14.4; and $p=0.02 ; 95 \%$ CI 1.2-8.1, respectively).

\section{DISCUSSION}

Approximately 15 million adult patients undergo noncardiac surgery each year in the United States. ${ }^{18}$ Cardiovascular morbidity and mortality account for most postoperative complications. ${ }^{19}$ In particular, in patients undergoing major vascular surgery, perioperative adverse cardiac outcomes are very high $(5-15 \%)$ and thus reflect the systemic nature of the atherosclerotic process with common risk factors between $\mathrm{CAD}$ and peripheral vascular disease. ${ }^{9}$ As a consequence, there exists a significant need for a simple, safe, and cost-effective method to 
accurately predict perioperative cardiac risk before noncardiac surgery.

Historically, many attempts to accurately stratify cardiac risk have been made. ${ }^{7,20,21}$ In these studies, history of $\mathrm{CAD}$, congestive heart failure, diabetes mellitus, and chronic renal failure all increased the risk of perioperative cardiac adverse events. In our experience, no parameter significantly affected perioperative cardiac mortality; however, age $>80$ years, chronic renal failure, congestive heart failure, and valvular heart disease affected cardiac morbidity. Only valvular heart disease demonstrated to be an independent risk factor for perioperative nonfatal myocardial infarction in patients undergoing abdominal aortic surgery.

In 1996, the American College of Cardiology/ American Heart Association (ACC/AHA) published the first clinical guidelines for preoperative cardiac risk stratification in patients undergoing noncardiac surgery. These guidelines were recently updated in 2007. ${ }^{9}$ Other societies published their own guidelines, ${ }^{22,23}$ and numerous discrepancies exist between them. Several authors reported controversial results with the use of these guideline recommendations and the cardiac risk indexes to improve perioperative adverse cardiac outcomes. ${ }^{10,24,25}$ In our experience, the use of a standardized preoperative cardiac algorithm with the selective use of stress testing suggests that patients with a positive stress test have a higher risk of cardiac complications in the perioperative period.

Most of the guidelines recommend the select use of supplemental preoperative noninvasive cardiac testing to provide an objective measure of cardiac functional capacity, to identify the presence of significant myocardial ischemia, and to evaluate the risk of perioperative cardiac adverse outcomes. ${ }^{9}$ In particular, cardiac stress testing has been advocated to assess patients before major vascular surgery. In contrast, several authors ${ }^{26-29}$ demonstrate that the positive predictive value of stress testing is very poor $(5-25 \%)$ and conclude that its use to predict perioperative adverse cardiac events is very limited. Our experience in aortic surgery partially supports these conclusions; in fact, in our population study a positive preoperative cardiac stress testing represents a risk factor for postoperative cardiac adverse events, although its positive predictive power remains still low (9/86 cases, $10.5 \%)$.

As a consequence, a positive preoperative noninvasive cardiac stress testing seems to propose the value of appropriate perioperative medical therapies to lower the cardiac risk rather than extensive use of myocardial revascularization. ACC/AHA 2007 guidelines 9 recommend beta-blockers should be given to patients undergoing major vascular surgery, such as open aortic surgery, owing to the finding of ischemia on preoperative noninvasive cardiac testing. The most recent Perioperative Ischemic Evaluation (POISE) trial ${ }^{12}$ minimized the benefits resulting in the assumption of betablocker medical therapy in the perioperative period; in fact, this large randomized trial demonstrated that beta-blocker therapy reduced cardiac deaths and complications at 30 days, but it increased the mortality from all causes and the cerebrovascular events with respect to placebo administration. In our preoperative cardiac assessment, beta-blocker medical therapy and a high level of postoperative care in ICU is the most used approach in patients with a poor cardiac functional capacity undergoing endovascular treatment. In selected cases, when an endovascular approach is not feasible, the consultant suggested a supplemental evaluation of the status of the coronary arteries with invasive procedures.

Controversial data exist regarding benefits of myocardial revascularization in reducing perioperative cardiac adverse events in patients undergoing major vascular surgery; ${ }^{9,13,14,30}$ ACC/AHA 2007 guidelines ${ }^{9}$ stated that coronary revascularization before noncardiac surgery is useful in patients with stable angina and significant left main coronary artery stenosis or a 3-vessel disease, or in patients with unstable angina. In our population study, more than of one-third of patients (34/86, $39.5 \%$ ) with positive preoperative noninvasive cardiac stress testing underwent coronary angiography; 13 patients were successfully treated and one of these developed a postoperative fatal myocardial infarction, whereas in the untreated group we did not record any cardiac deaths. Overall, $<4 \%$ $(13 / 531)$ of the patients underwent preoperative coronary revascularization before their vascular surgical procedure.

The need for minimizing perioperative risks has led to the broad application of endovascular techniques for AAA management. Endovascular techniques allow the treatment of patients whose comorbidities make conventional open repair difficult or high risk. ${ }^{31}$ For this reason, patients with a positive stress test could be treated with endovascular treatment when anatomically feasible. In our experience, endovascular treatment reduced perioperative cardiac morbidity rates compared with patients undergoing standard open surgical repair $(3.9 \%$ vs. $9.1 \% ; p=0.01)$. This should encourage many surgeons to stress the indications to endovascular treatment in this subgroup of 
patients so as to decrease cardiac morbidity in the perioperative period.

\section{CONCLUSIONS}

In our experience, the use of an accurate preoperative cardiac assessment allowed us to obtain satisfactory perioperative results in patients undergoing abdominal aortic surgery. Valvular heart disease seems to be an independent factor predictive of perioperative cardiac complications. A positive noninvasive stress testing represents a strong independent predictive factor of cardiac complications in the perioperative period. These patients could benefit from a less invasive interventional approach, such as endovascular treatment.

\section{REFERENCES}

1. Mackey WC, O'Donnell TFJ, Callow AD. Cardiac risk in patients undergoing carotid endoarterectomy: impact on perioperative and long-term mortality. J Vasc Surg 1990;11:226-233.

2. Krupski WC, Layug EL, Reilly LM, et al. Comparison of cardiac morbidity rates between aortic and infrainguinal operations: two-year follow-up. Study of Perioperative Ischemia Research Group. J Vasc Surg 1993;18:609-615.

3. L'Italien GJ, Cambria RP, Cutler BS, et al. Comparative early and late cardiac morbidity among patients requiring different vascular surgery procedures. J Vasc Surg 1995;21:935-944.

4. Devereaux PJ, Goldman L, Yusuf S, et al. Surveillance and prevention of major perioperative ischemic cardiac events in patients undergoing noncardiac surgery: a review. CMAJ 2005; 173:779-788.

5. Aronow WS, Ahn C. Prevalence of coexistence of coronary artery disease, peripheral arterial disease, and atherothrombotic brain infarction in man and women $>62$ years of age. Am J Cardiol 1994;74:64-65.

6. Sukhija R, Aronow WS, Yalamanchili K, et al. Prevalence of coronary artery disease, lower extremity peripheral arterial disease, and cerebrovascular disease in 110 men with an abdominal aortic aneurysm. Am J Cardiol 2004;94: 1358-1359.

7. Lee TH, Marcantonio ER, Mangione CM, et al. Derivation and prospective validation of a simple index for prediction of cardiac risk of major noncardiac surgery. Circulation 1999;100:1043-1049.

8. Kertai MD, Boersma E, Bax JJ, et al. Comparison between serum creatinine and creatinine clearance for the prediction of postoperative mortality in patients undergoing major vascular surgery. Clin Nephrol 2003;59:17-23.

9. Fleisher LA, Beckman JA, Brown KA, et al. ACC/AHA 2007 guidelines on perioperative cardiovascular evaluation and care for noncardiac surgery: a report of the American College of Cardiology/American Heart Association Task Force on Practice Guidelines. Circulation 2007;116:e418-e499.

10. Falcone RA, Nass C, Jermyn R, et al. The value of preoperative pharmacologic stress testing before vascular surgery using ACC/AHA guidelines: a prospective, randomized trial. J Cardiothorac Vasc Anesth 2003;17:694-698.
11. Lindenauer PK, Pekow P, Wang K, et al. Perioperative betablocker therapy and mortality after major noncardiac surgery. N Engl J Med 2005;353:349-361.

12. Deveraux PJ, Yang H, Yusuf F, et al; on behalf of POISE Study Group. Effects of extended-release metoprolol succinate in patients undergoing non-cardiac surgery (POISE trial): a randomised controlled trial. Lancet 2008;371: 1839-1847.

13. McFalls EO, Ward HB, Moritz TE, et al. Coronary-artery revascularization before elective major vascular surgery. N Engl J Med 2004;351:2795-2804.

14. Godet G, Riou B, Bertrand M, et al. Does preoperative coronary angioplasty improve perioperative cardiac outcome? Anesthesiology 2005;102:739-746.

15. Qaseem A, Snow V, Fitterman N, et al. Risk assessment for and strategies to reduce perioperative pulmonary complications for patients undergoing noncardiothoracic surgery: a guidelines from the American College of Physicians. Ann Intern Med 2006;144:575-580.

16. Labib SB, Goldstein M, Kinnunen PM, Schick EC. Cardiac events in patients with negative maximal versus negative submaximal dobutamine echocardiograms undergoing noncardiac surgery: importance of resting wall motion abnormalities. J Am Coll Cardiol 2004;44:82-87.

17. Etchells E, Meade M, Tomlinson G, Cook D. Semiquantitative dipyridamole myocardial stress perfusion imaging for cardiac risk assessment before noncardiac vascular surgery: a meta-analysis. J Vasc Surg 2002;36:534-540.

18. DeFrances CJ, Hall MJ. 2002 National Hospital Discharge Survey. Adv Data 2004;342:1-29.

19. Eagle KA, Rihal CS, Mickel MC, et al. For the CASS investigators and University of Michigan Heart Care program. Cardiac risk of noncardiac surgery: influence of coronary disease and type of surgery in 3368 operations. Circulation 1997;96:1882-1887.

20. Goldman L, Caldera DL, Nussbaum SR, et al. Multifactorial index of cardiac risk in noncardiac surgical procedures. $\mathrm{N}$ Engl J Med 1977;297:845-850.

21. Detsky AS, Abrams HB, McLaughlin JR, et al. Predicting cardiac complications in patients undergoing noncardiac surgery. J Gen Intern Med 1986;1:211-219.

22. Snow V, Barry P, Fihn SD, et al. Evaluation of primary care patients with chronic stable angina: guidelines from the American College of Physicians. Ann Intern Med 2004; 141:57-64.

23. Snow V, Barry P, Fihn SD, et al. Primary care management of chronic stable angina and asymptomatic suspected or known coronary artery disease: a clinical practice guideline from the American College of Physicians. Ann Intern Med 2004; 141:562-567.

24. Gordon AJ, Macpherson DS. Guideline chaos: conflicting recommendations for preoperative cardiac assessment. Am J Cardiol 2003;91:1299-1303.

25. Legner VJ, Doerner D, McCormick WC, et al. Clinician Agreement with perioperative cardiovascular evaluation guidelines and clinical outcomes. Am J Cardiol 2006;97: 118-122.

26. Urbinati S, Di Pasquale G, Andreoli A, et al. Preoperative non-invasive coronary risk stratification in candidates for carotid endarterectomy. Stroke 1994;25:2022-2027.

27. Baron JF, Mundler O, Bertrand M, et al. Dipyridamole-thallium scintigraphy and gated radionuclide angiography to assess cardiac risk before abdominal aortic surgery. N Engl J Med 1994;330:663-669. 
28. Leppo J, Plaja J, Gionet M, et al. Noninvasive evaluation of cardiac risk before elective vascular surgery. J Am Coll Cardiol 1987;9:269-276.

29. Hanson $\mathrm{P}$, Pease $\mathrm{M}$, Berkoff $\mathrm{H}$, et al. Arm exercise testing for coronary artery disease in patients with peripheral vascular disease. Clin Cardiol 1988;11:70-74.

30. Landesberg G, Mosseri M, Shatz V, et al. Cardiac troponin after major vascular surgery: the role of perioperative ischemia, preoperative thallium scanning, and coronary revascularization. J Am Coll Cardiol 2004;44:569-575.

31. Greenhalgh RM, Brown LC, Kwong GP, et al. Comparison of endovascular aneurysm repair with open repair in patients with abdominal aortic aneurysm (EVAR trial 1), 30-day operative mortality results: randomised controlled trial. Lancet 2004;364:843-848. 